\title{
Development and Suggestions on Container Twist Lock Handling in Automated Container Terminals
}

\author{
Panpan Zhang ${ }^{1,2, *}$ \\ 1 China Waterborne Transport Research Institute, Beijing, China \\ ${ }^{2}$ Key Laboratory of Logistics Equipment \& Control Engineering, Beijing, China
}

\begin{abstract}
In this paper automated container terminals were introduced and characteristics of the automated container terminals were summarized. Besides, a common problem of twist lock handling technology in automated container terminal was proposed. different kinds of automatic twist lock handling technologies and equipment were collected and analyzed. Finally, suggestions on twist lock handling were given. With demands of automated container terminals, the twist lock handling technology will still rapidly develop in future.
\end{abstract}

\section{Introduction}

Container transportation is a modern advanced transportation mode. Ship transportation, as a main mode of container transportation, has developed rapidly. In recent years, the United States, Britain, Japan and other countries import and about $70 \%$ to $90 \%$ of cargo exported aboard takes advantage of container transportation. The Container terminal shown in Figure 1 is the hub of land-water inter-modal transportation. container terminal plays an important role in the whole world trade process.

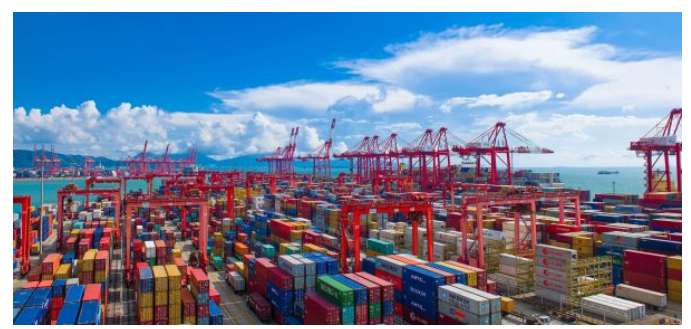

Figure 1. The container terminal

Container handling in terminals is usually consisted of ship handling, horizontal transport and yard handling as shown in Fig. 2. Since the birth of the container terminal, the pace of automation has never stopped. Globally, the automation process of container terminals is accelerating.

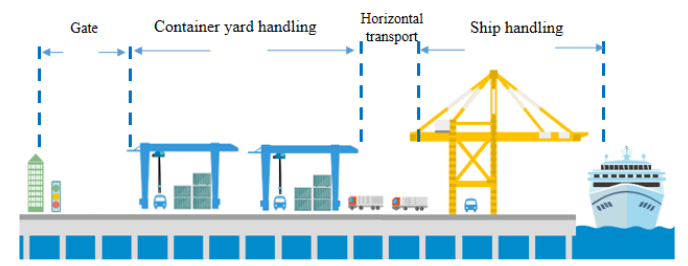

Figure 2. Typical composition of a container terminal
In this paper automated container terminals were introduced and characteristics of the automated container terminals were summarized. Besides, a common problem of twist lock handling in automated container terminal was proposed. Different kinds of automatic twist lock handling technologies and equipment were collected and analyzed. Finally, suggestions on development of twist lock handling technology were given.

\section{Characteristics of the automated container terminals}

With fast development of the global economy, the maritime container volume around the world shows a growing trend. Container ships are becoming larger and larger. Container handling with stable, efficient, energysaving, environmental protection and low cost has become the focus of attention of container terminals.

In automated container terminals, ship handling, horizontal transport and yard handling processes can run as the terminal operation system are set. Since the first fully automated container terminal was put into commercial operation in 1993, automated container terminals have developed rapidly. ECT terminal and Euromax terminal at Rotterdam Port, CTA terminal at Hamburg Port, and automated terminal at Qingdao Port as shown in Figure 3 have all become milestones in the development history of fully automated container terminals, witnessing technological changes in container terminal ship handling, horizontal transportation, yard handling and so on. According to incomplete statistics, 10 fully automated container terminals have been built worldwide.

\footnotetext{
* Corresponding author: zhangpanpan@wti.ac.cn
} 


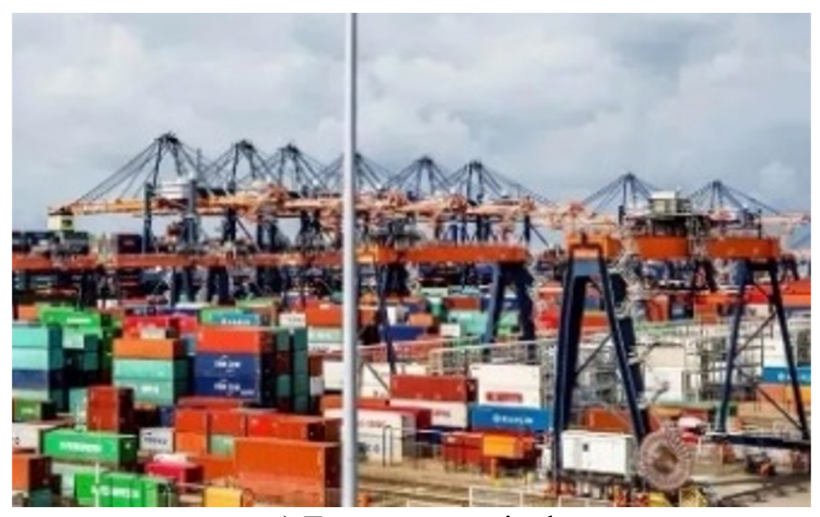

a) Euromax terminal

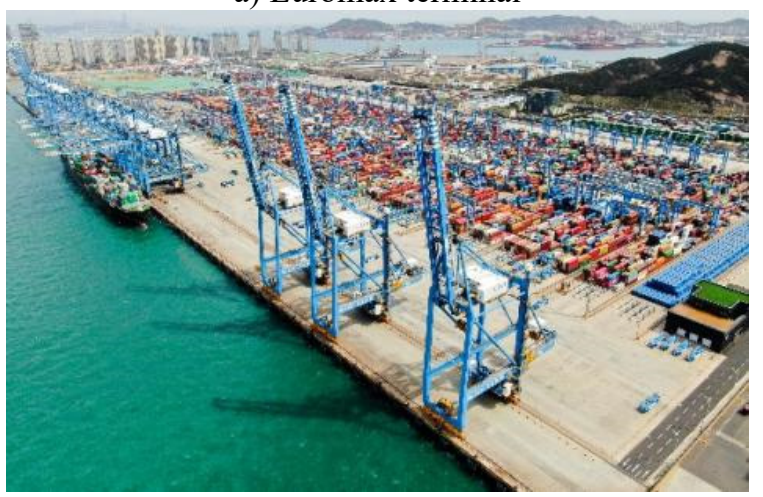

b) Qingdao Qianwan terminal

Figure 3. Typical examples of Automated container terminal

1) Automated container terminals play an important role in reducing the labor cost improving the capacity of the port, reducing the energy consumption of the loading and unloading operation. It is an inevitable trend of port development [1]. Characteristics of automated container terminals can be summarized as follows.

2) high reliability:container operations in automated terminal can avoid influence of human factors and can improve the reliability of the operation;

high operation safety: in automated container terminals, all operations were carried out as setting $\mathrm{s}$ in terminal system, which results in higher operation safety and is conducive to building safe ports;

3) lower labor costs: automatic operation can reduce the number of personnel assigned to the terminal which is good to reduce labor costs;

4) improvement of the working environment: greatly improve the working environment and reduce labor intensity, reduce occupational diseases, flexible deployment of personnel, shift convenience;

5) low energy consumption and low emission: the extensive use of all-electric drive equipment and the application of energy feedback technology can effectively reduce the energy consumption of port operations and can improve the port environment;

6) larger initial investment: compared with the traditional container terminal, the investment of automatic handling equipment, yard foundation and terminal operation system are higher;

\section{Development of Twist lock handling technology}

Handling of container twist lock is an important link in container handling process and it is still a bottleneck in automated container terminal. At present, there are many ways of container locking and unlocking in the world, but manual installation and disassembly are adopted as shown in Fig. 4 [2].

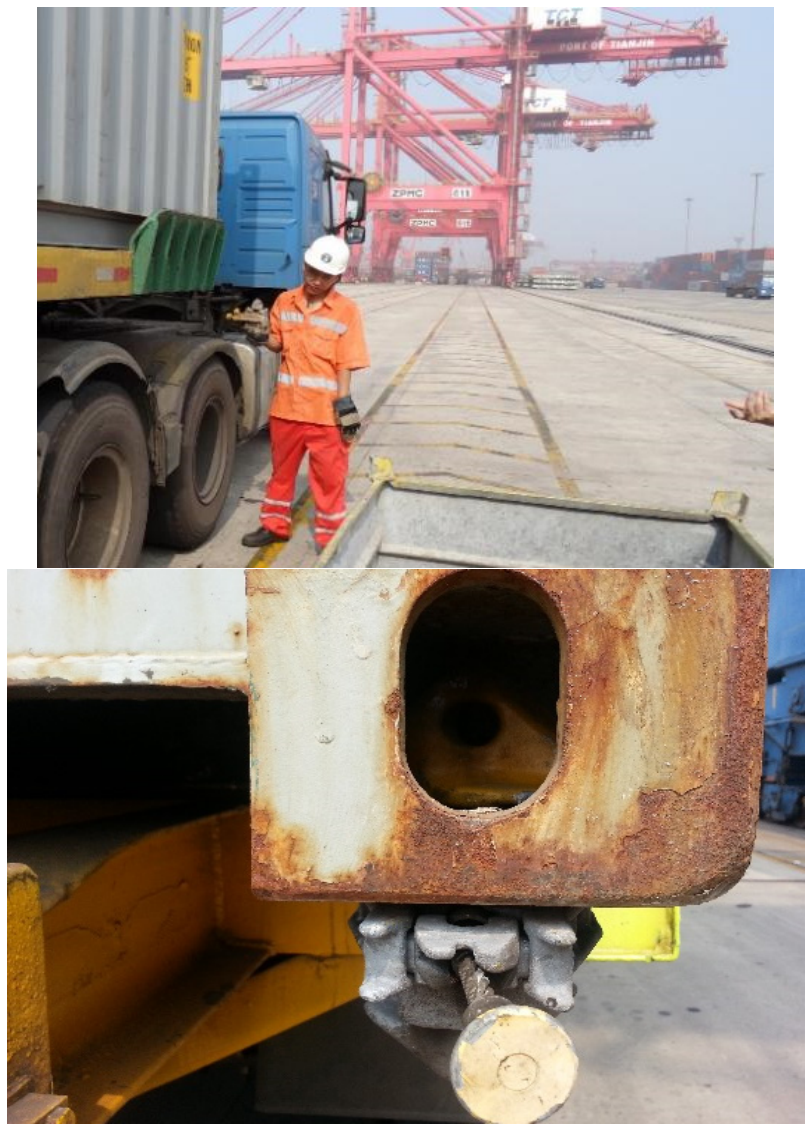

Figure 4. Manual handling of twist lock

During ship handling operations, a container vessel with a length of more than 200 meters requires at least 6 to 10 workers in the removal of container locks which seriously restricts the automation of container handling process. Therefore, many research institutions and enterprises in China and abroad have made some progress in areas of twist lock handling. In this part, the research progress of twist lock handling technology at container terminals were comprehensively introduced and technical characteristics were analyzed.

Kalp released a fully autonomous lashing platform as shown in Fig. 5. The platform adopts hydraulic system and the success rate of twist lock handling is more than $95 \%$ [3]. The biggest feature of the platform is that twist locks can be removed or installed under different working conditions. When the twist lock is removed, the twist lock is automatically placed in the internal collector. 2,400 twist locks can accommodated in each collector. Besides, the platform is adjustable for all kinds of standard containers. 


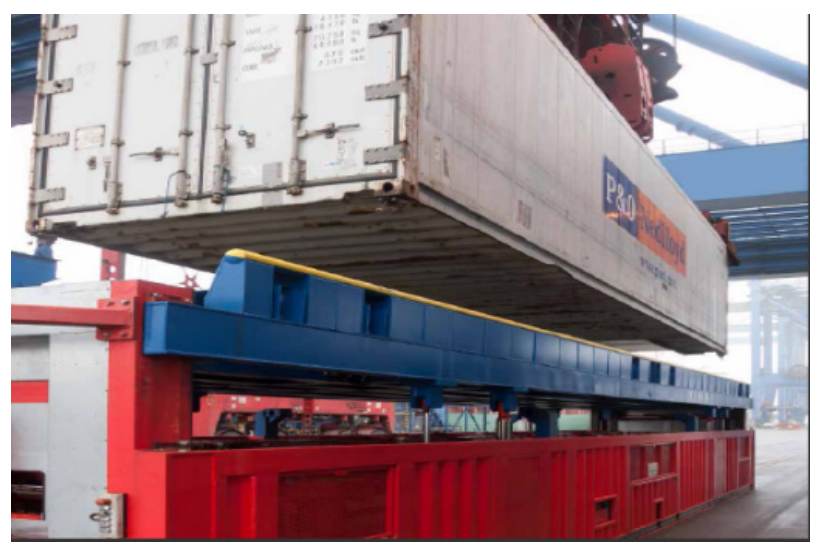

Figure 5. The autonomous lashing platform

The system can be operated smoothly by integrated hydraulic shock absorption technology as shown in Fig. 6. the design can absorb the force and reduce the impact on cargo and equipment [2]. The energy needed to operate the twist lock handling is generated by the gravity of the container itself. The device is efficient and does not rely on external power (such as fuel and electricity), thus maximizing flexibility for operations.

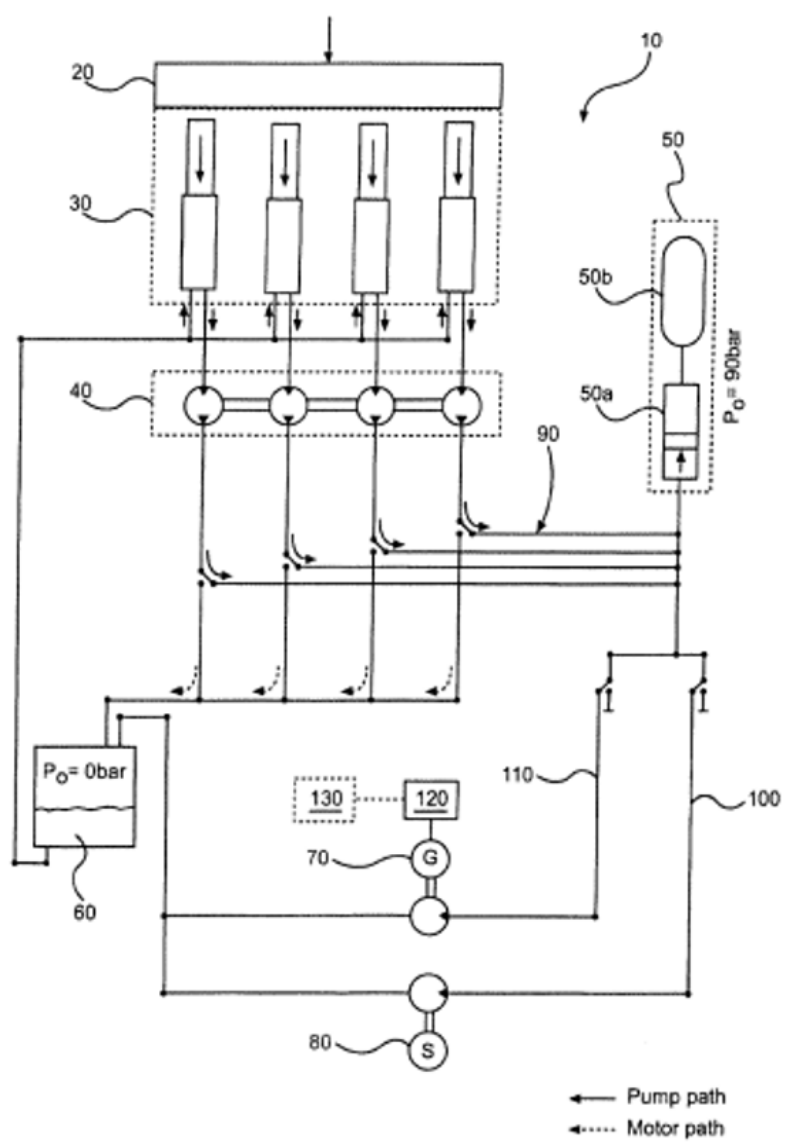

Figure 6. Diagram of the hydraulic system

C. Liang et al. From Nanyang Technological University developed a Grizzly unlocking experimental system based on a manipulator [4]. The system is mainly composed of a manipulator, claws, a camera and a mobile platform. Based on visual recognition, the platform can obtain the position and can recognize the type of the twist lock through the camera. and then the twist lock through the manipulator and three movable claws can be removed. The equipment still needs to be improved to adapt to the on-site situation in the busy horizontal transport conditions of container terminals.

Liu et al. from Qingdao Qianwan Container Terminal proposed an automatic disassembly design for container twist locks [5]. Base on the design, a robotic twist lock handling system were developed as shown in Fig. 7. The system is consisted of six industrial robots, a conveyor line and collecting box. Each robot is equipped with visual recognition and sensors. The optical fiber sensor is used to detect the movement of twist locks and the cylinder. The position of twist locks are determined by $\mathrm{CCD}$ and $\mathrm{Z}$ direction position sensor, and then the signal is transmitted to the robot controller through image processing to correct the deviation of the unloading position. The twist lock can be removed from the container through the servo torque control. The conveyor line connects the working positions of the six robots to achieve the collecting and distribution of the lock.

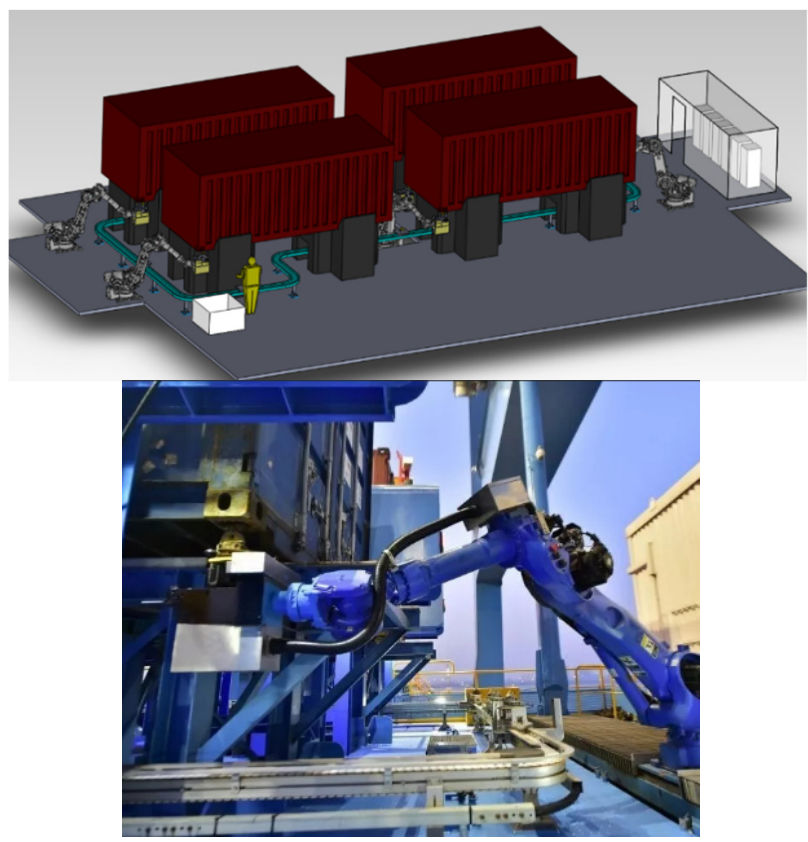

Figure 7. Robotic lock removal device and system flow chart of Xinqianwan Container Terminal

Research in the field of automatic twist lock handling was also carried out by China Waterborne Transport Research Institute [6]. An experimental prototype of the automatic handling actuator was designed and manufactured as shown in Fig. 8. The experimental prototype can realize the wireless control of the unlocking device through the controller, and realize the automatic unlocking, manual unlocking and fault feedback and processing. The unlocking time of the automatic unlocking execution device is not more than 5 seconds. Interlocking protection, fault self-diagnosis, warning and treatment measures were integrated in the control system as well as in the remote panel as shown in Fig.9 . 


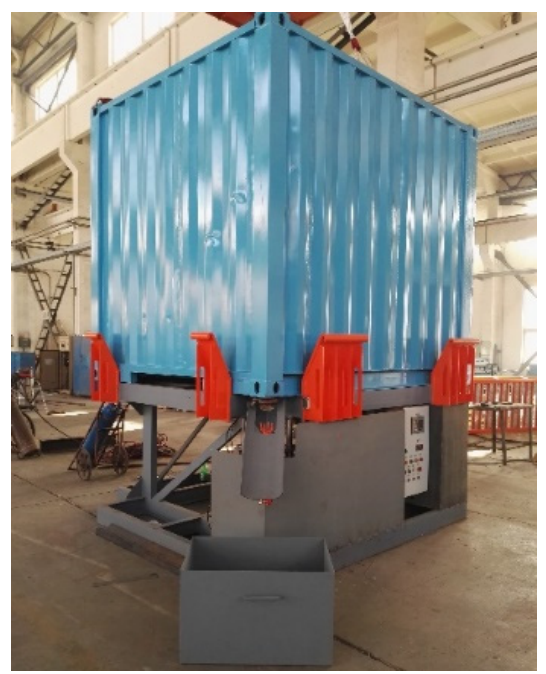

Figure 8. The automatic handling prototype

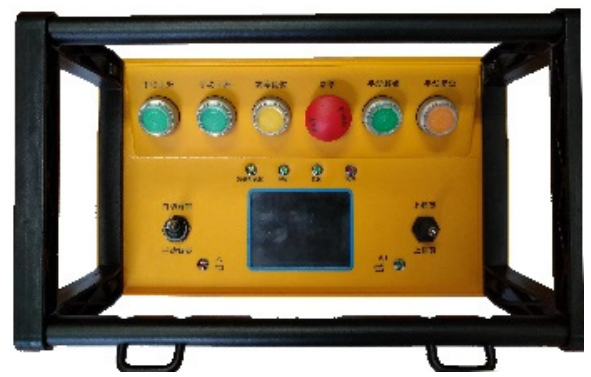

Figure 9. The remote control panel

The principle of automatic twist lock handling devices mentioned above are similar and a series of industrial tests were carried out. However, the automatic twist lock handling system still can not suitable for all kinds of twist locks. Thus, there are suggestions on twist lock handling in the following part.

1) it is necessary to improve standardization of twist locks. There are regulation on the mechanical properties of twist locks in Standard of ISO1161 and there is no mandatory requirement for the shape of twist locks. This results in a wide variety of twist locks, making the automatic twist lock handling difficult. The standardization of twist locks is the basis for the comprehensive upgrading of automatic twist lock handling technology.

2) with development of image recognition technology and flexible robot technology, the twist lock handling device will be more intelligent. The twist lock handling device currently developed mainly make use of multi-joint industrial robots. In future flexible robots may bring new opportunities to the twist lock handling area.

3 ) it is necessary to increase the automation degree of twist locks. If the twist lock is automated, handling of twist locks in container vessels will be labor-saving. It is an important aspect of the automated container terminal.

\section{Conclusion}

With development of global trade, automated container terminals are booming in recent years.In this paper automated container terminals were introduced.
Characteristics of automated container terminals were analyzed and summarized. Besides, a common problem of twist lock handling in automated container terminals was proposed. In the automatic twist lock handling area, different kinds of technologies and equipment were collected and analyzed. Finally, suggestions on twist lock handling development were given. With demands of automated container terminals, twist lock handling technology will still develop in future. By solving automation of the twist lock handling process, the potential ability of automated container terminals can be taken into a new stage.

\section{References}

1. He Jihong, Application Status and Prospect of Automatic Container Terminal Handling Process System,Port \& Waterway Engineering, 2018(6):5-8.

2. Xie Chen, Zhang Panpan, Fei Haibo.Development Trend of Container Twist Locks Technology. China Water Transport, 2015(7):4-7.

3. Rainer Kapelski, fully autonomous lashing platform US Patent No.US9499336,2016

4. Zhang, L., et al.: Twist-lock pose estimation and grasping based on CAD model. In: Proceedings of the 3rd IFTOMM International Symposium on Robotics and Mechatronics, ISRM2013, Singapore, pp. 739747 (2013)

5. Panpan Zhang, Chen Xie, Haibo Fei. Twist Lock Unlocking Process Research and Unlocking Fixture Design in Container Terminals, Advances in Engineering Research (2015):1122-1126.

6. Liu Yang, Zhang Wei, Zhuang Xinchuan. Design of automatic disassembly device for container twist lock, Port Science \& Technology. 2012, 10:27-18. 